\title{
Lightweight Robotic Arm Actuated by Shape Memory Alloy (SMA) Wires
}

\author{
Serket Quintanar-Guzmán, Somasundar Kannan, Miguel A. Olivares-Mendez, Holger Voos \\ Interdisciplinary Centre for Security, Reliability and Trust \\ University of Luxembourg \\ Luxembourg, Luxembourg \\ Email: serket.quintanar@uni.lu
}

\begin{abstract}
The current paper discusses the design, modeling and control of a Light weight robotic arm actuated by Shape Memory Alloy (SMA) actuators, usable for applications such as Aerial Manipulator. Compared to servo motor based robotic arm the proposed design has an added advantage of light weight and high force to mass ratio, but further introduces the problem of nonlinearities such as Hysteresis into the system. A nonlinear dynamic model of the hysteretic robotic arm is systematically developed to perform closed loop simulations. A Joint Space control is performed using Variable Structure Control and the closed loop performance is successfully verified by simulation studies.
\end{abstract}

Keywords-Light weight robotic arm, aerial manipulator, Shape Memory Alloy (SMA), joint space control

\section{INTRODUCTION}

The design and development of Light Weight Robotic arms have attracted more attention recently in the context of Aerial Applications. By attaching a robotic arm one can increase the capability of aerial vehicles such as quadrotors or vehicles with hovering capabilities. Aerial Manipulators that is an Aerial Vehicle attached with a manipulator can transform an Unmanned Aerial Vehicle (UAV) from passive sensor to an active actuator by performing tasks such as object manipulation, assembly, transportation etc. The limited availability of payload on Micro Aerial Vehicle has motivated the robotic community further study the problem [1]-[3].

Let us continue here briefly with the existing literature. In [1], [4] the authors had designed and controlled a hyperredundant manipulator for Mobile Manipulating Unmanned Aerial Vehicles. Here the objective was use the redundancy of the manipulator to minimize the influence of the manipulator on the stability of the Aerial Vehicle. Here at least 9 servo joints were used which lead to a total weight of approximately $1.3 \mathrm{Kg}$. In [5] the authors discussed the mechanical design of a 6 degree-of-freedom (DOF)aerial manipulator for the purpose of assembly using UAVs. Here they used commercially available servos for the actuation which lead to a total weight of $1.5 \mathrm{Kg}$. In [6] a light weight compliant 2 DOF robotic arm was developed using linear servos where the total weight of the arm was about 325 grams. In [7] the authors modeled and controlled a 3D printed 5-DOF light weight robot arm which had lead to a total weight of 250 grams.
With respect to the above contributions the current paper proposes a light weight robotic arm actuated by Shape Memory Alloy (SMA) wires which weighs only 48 grams. SMA actuators, which can be categorized under smart material systems with Shape Memory Effect (SME), have special characteristics like high force to mass ratio, small size, noiseless operation, and bio-compatibility which makes them a great alternative to conventional hydraulic, electric and pneumatic actuators. In spite of all its advantages they are still slow and highly nonlinear systems with Hysteresis problem, which is challenging to perform precise control operation. SMA wires are being used in applications from medical implants like inter-arterial supports [8], [9], dental applications [10], car mirror actuators [11] or SMA based motors [12], and robotic manipulators as arms, hands or robotic fingers [13]-[15] to general purpose actuators [16]-[21].

The key contributions of this article can be categorized into two. The first contribution include Mechanical design and modeling of a light weight robotic arm based on an existing SMA joint actuator [18]. Secondly the contributions include application of a Nonlinear control technique to perform simulation studies on a Nonlinear Manipulator with hysteresis problem. The reminder of the paper is organized as follows. First we describe the SMA based robot arm design including SMA actuation and mechanical design. This is followed by modeling of SMA based robotic arm and the independent joint space control. Finally the simulation results and conclusions are presented.

\section{Shape Memory Alloy (SMA) based Robot Arm DESIGN}

The robotic arm presented here consists of two links with one degree of freedom (DOF) actuated by two antagonistic SMA wires. The main goal of this work is to design and develop a light-weight robotic arm to be later used with robotic aerial vehicles, since it is a great challenge to make an optimal use of available payload of an aerial vehicle such as a quadcopter. We propose a light-weight design, which enables the arm to be implemented without significantly decreasing the quadcopter's available payload. Here we will first discuss the concept of SMA based actuation and then the mechanical design of robotic arm. 


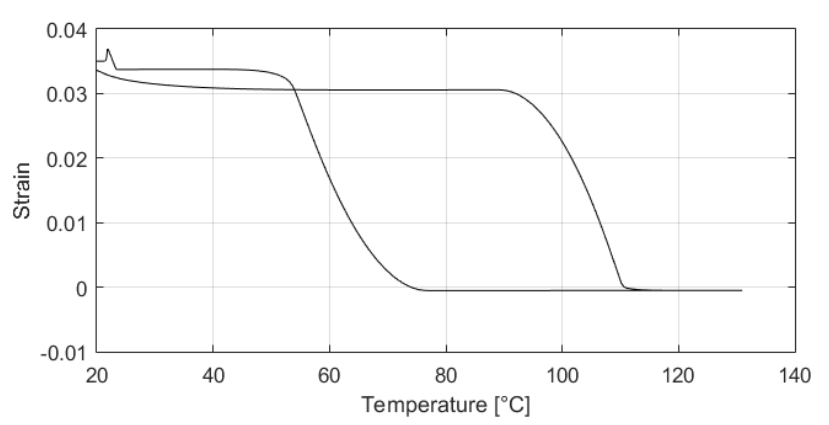

Fig. 1. System Hysteresis Curve Temperature-Strain

\section{A. SMA based Actuation}

The SMA are a group of metallic alloys with Shape Memory Effect (SME). This phenomenon is the ability of the material to recover its original shape after being deformed when subject to the appropriate thermal procedure. This effect is caused by a transformation of the material's crystalline structure. The most common types of SMA are the Niquel-Titanium SMA wires (NiTi wires). When the NiTi wire is at lower temperature (martensite state) it can be deformed with a relatively low stress. When heated, a phase change occurs and the material transforms to austenite state and recovers its original form and size. The capability to recover form and shape supports their usage in actuation but at the same time introduces significant challenges due to the presence of nonlinearity such as hysteresis. The hysteresis present in our current light weight robotic arm can be seen in Fig. 1. Here we can see the presence of a double loop hysteresis due to the interaction of the antagonistic SMA wires.

\section{B. Mechanical Design}

A Computer Aided Design (CAD) model of the proposed robotic arm is shown in Fig. 2. The robotic arm is actuated by two antagonistic SMA wires. These wires apply force over a mechanical joint couple with a torsion spring, this coupler was proposed in [18]. The end effector is attached to the Coupler 1 (Fig. 2) by a $15 \mathrm{~cm}$ long carbon fiber link. This coupler is joined trough a torsion spring to the Coupler 2 (see Fig. 2), that allows to control the overall stiffness of the mechanical system by adjusting the position of the second coupler so the force of the spring adapts as necessary. The winding wheels are a winding mechanism for the SMA wires, which enables the use of longer SMA wires for larger movement range without increasing the size of the links.

The end effector is actuated by a third SMA wire with bias spring, so the model is completely motor-free. The design is planned to be constructed partially by 3D printing and partially by custom made carbon fiber parts, achieving a total weight of $48 \mathrm{~g}$. In spite of the light-weight characteristic, the arm is capable of lifting a load up to $500 \mathrm{~g}$, and can be increased by implementing thicker SMA wires. This amount of load is not far from the limit payload of a small quadcopter.

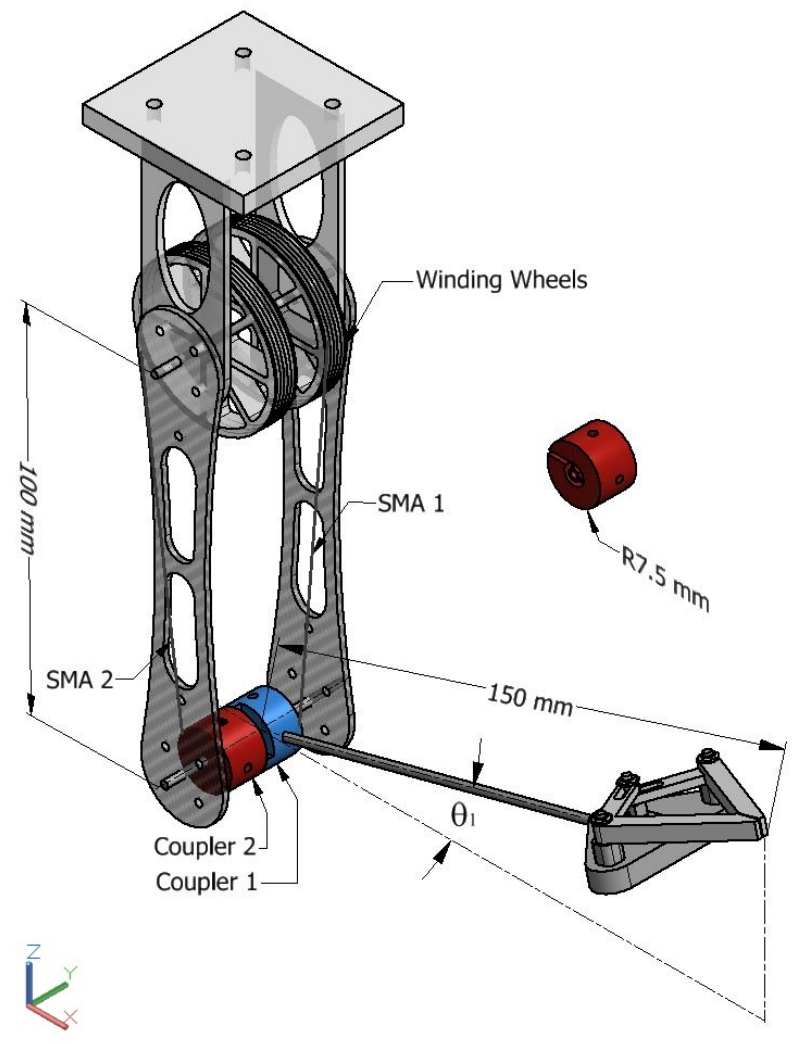

Fig. 2. Proposed Robot Arm CAD Model

\section{System Modeling}

The robotic arm proposed by our team was modeled by two highly coupled subsystems: the SMA wires model and the dynamics of the robotic arm. This two subsystem are related trough the kinematic model as shown in Fig. 3. The SMA wires model is further divided into three more subsystems: thermal dynamics, phase transformations and constitutive model. The SMA wire model was proposed in [17] and adapted to fit the characteristic of dynamic stress of an antagonistic SMA wire actuator. Fig. 4 shows the block diagram of the SMA wire model. Each sub-model will be explained in more detail in the next sub-sections.

\section{A. Heat Transfer Model}

The heat transfer model consists of natural convection for cooling and electrical heating by Joule effect [17]:

$$
m_{w} c_{p} \frac{d T}{d t}=\frac{V^{2}}{R}-h A_{w}\left(T-T_{a m b}\right)
$$

where $V$ is the voltage, $R$ is the electric resistance per unit length, $c_{p}$ is the specific heat, $m_{w}$ is the mass per unit length, $A_{w}$ is the wire surface area, $T_{a m b}$ the ambient temperature and $T$ the SMA wire temperature. The heat convection factor $h$ is approximated by a second order polynomial of the temperature as:

$$
h=h_{0}+h_{2} T^{2}
$$




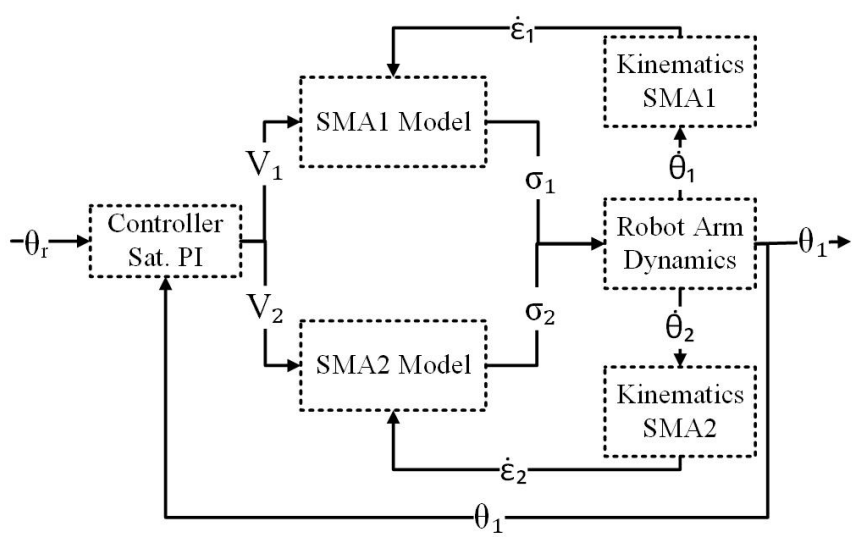

Fig. 3. Complete System Block Diagram

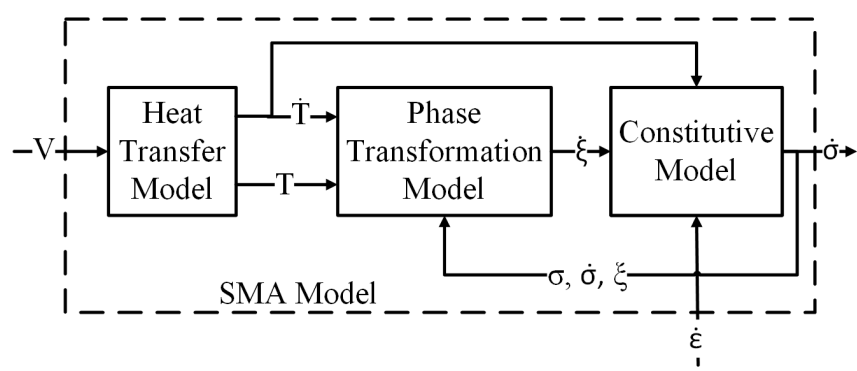

Fig. 4. SMA Wire Block Diagram

\section{B. SMA Wire Phase Transformation Model}

The phase transformation model computes the SMA wires martensite fraction $(\xi)$. This value depends directly of the temperature rate sign. Since the SMA wire is a highly hysteretic system, this sub-model consists of two equations which describe the cooling and heating curve of the Martensite fraction. When heating, the phase transformation from martensite to austenite is given by

$$
\xi=\frac{\xi_{M}}{2}\left\{\cos \left[a_{A}\left(T-A_{s}\right)+b_{A} \sigma\right]+1\right\}
$$

for $A_{s}+\frac{\sigma}{C_{A}} \leq T \leq A_{f}+\frac{\sigma}{C_{A}}$

During cooling, the phase transformation from austenite to Martensite is given by

$$
\xi=\frac{1-\xi_{A}}{2} \cos \left[a_{M}\left(T-M_{F}\right)+b_{M} \sigma\right]+\frac{1+\xi_{A}}{2}
$$

for $M_{s}+\frac{\sigma}{C_{M}} \leq T \leq M_{f}+\frac{\sigma}{C_{M}}$

where $M_{s}, M_{f}, A_{s}, A_{f}$ are the start and end transformation temperatures for martensite and austenite transformation respectively. And $a_{A}=\frac{\pi}{\left(A_{f}-A_{s}\right)}, a_{M}=\frac{\pi}{\left(M_{s}-M_{f}\right)}$, $b_{A}=-\frac{a_{A}}{C_{A}}, b_{M}=-\frac{a_{M}}{C_{M}}, C_{A}$ and $C_{M}$ are curve fitting parameters.

\section{Wire Constitutive Model}

This model was first proposed in [22] and then modified in [17]. The wire constitutive model describes the relation between stress $\sigma$, strain $\varepsilon$, temperature $T$ and martensite fraction $\xi$. The constitutive model is written as:

$$
\dot{\sigma}=E \dot{\varepsilon}+\Omega \dot{\xi}+\Theta \dot{T} .
$$

Here $\Omega$ and $\Theta$ represent the thermal expansion coefficient and phase transformation constant respectively. The Young's modulus $E$ was adapted to meet the dynamic stress characteristic of antagonistic SMA wire

$$
E=\xi E_{M}+(1-\xi) E_{A},
$$

where the sub-index indicates $M$ for martensite and $A$ for austenite. And

$$
\Omega=-E \varepsilon_{0}
$$

where $\varepsilon_{0}$ is the initial strain.

\section{Kinematic and Dynamic Model}

1) Kinematic Model: This model relates the SMA wire model with the dynamics of the robotic arm. The SMA wires strain is directly proportional to the angular position of the robotic arm. They are related kinematically as

$$
\dot{\varepsilon_{i}}=-\frac{r_{i} \dot{\theta}_{i}}{l_{0 i}}
$$

where $r_{i}$ is the respective coupler radius, $l_{0}$ the initial length of each wire and $\dot{\theta}_{i}$ the angular velocity of each coupler.

2) Dynamics: The dynamic model describes the dynamic behavior of the robotic arm mechanism. This model shows the relation between couplers, applied external forces (torsion spring and SMA wires) and load effects. The mechanical system dynamic behavior can be described by the follow general dynamic model:

$$
J \ddot{\theta}=\left[\begin{array}{c}
\tau_{w 1}(\sigma)-\tau_{s}(\theta)-\tau_{g}(\theta)-\tau_{\text {load }}(\theta)-b_{1} \dot{\theta}_{1} \\
-\tau_{w 2}(\sigma)+\tau_{s}(\theta)-b_{2} \dot{\theta}_{2}
\end{array}\right]
$$

where $b$ is the friction of the couplers and $\tau$ is the torque applied over the mechanical system by the SMA wires (w), the torsion spring (s), the weight of the gripper $(\mathrm{g})$ and the load. This model was developed in two parts: First the couplers mechanism, gripper, load and links dynamics and second the mathematical model of $\tau_{w}$ and $\tau_{s}$. The first part of the dyanamics was directly obtained from the CAD design shown in Fig. 2 developed in Autodesk/Inventor environment. The use of this approach brings great advantages as the automatic inclusion of the exact geometry of the pieces, masses, inertias and centers of mass, which are critical parameters for the dynamic analysis. The CAD model is imported via the SimMechanics toolbox in order to obtain a continuous dynamic MATLAB/Simulink model of the mechanical system.

The second part of the dynamics was derived from basic physical laws. The SMA wire force is inversely proportional 
to the stress $(\sigma)$ which can be calculated by integration of Eq. (5). Then the torque can be compute as follows:

$$
\tau_{w i}=F_{w i} r_{i}=A \sigma_{i} r_{i}
$$

where $r$ is the coupler radius and $A$ the transversal area of the wire. The torsion spring torque $\tau_{s}$ is calculated as

$$
\tau_{s}=k_{s}\left(\theta_{1}-\theta_{2}\right)+b_{s}\left(\dot{\theta}_{1}-\dot{\theta}_{2}\right)
$$

where $k_{s}$ is the spring constant and $b_{s}$ is the spring's friction factor, $\theta_{1}$ and $\theta_{2}$ are the angular position of each coupler respect to $X$-axis.

\section{JoInt Space Control}

The joint space control regulates the angular position of the end effector, which is attached to the coupler 1 (see Fig. 2 ) so the controlled variable is the angle of coupler 1 with respect to the $X$-axis $\left(\theta_{1}\right)$. For this regulation problem a Variable Structure Control (VSC) is implemented. This is a switching control law and is designed to force the plant's state trajectory onto a user-specified surface and maintain it on that surface. This control approach allows robustness to parameter uncertainties, nonlinearity of the model and external disturbances. The control law will switch depending on the state is over or under the user define surface. The VSC control law is given by

$$
s_{i}=c_{i}+c_{p i} e+c_{I i} \int e d t
$$

where $c_{p i}$ is the proportional gain, $c_{I i}$ is the integral gain, $c_{i}$ will preheat the wires and keeps it in tension when the error is zero. The error is defined as

$$
e=\theta_{1}-\theta_{r}
$$

The control signal (voltage) switching law is

$$
v_{i}=\left\{\begin{array}{l}
V_{i H}, s_{i} \geq \phi_{i} \\
s_{i}, 0 \leq s_{i}<\phi_{i} \\
0, s_{i}<0
\end{array}\right.
$$

here $\phi_{i}$ is the boundary layer and $V_{i H}$ is the maximum voltage, selected to protect the wires from overheating.

\section{Simulation Results}

The proposed SMA based robotic arm closed-loop control was evaluated only via simulation using Simulink/MATLAB. In order to conduct a closed-loop analysis an angular position tracking test was performed. The sinusoidal response was tested with a $0.016 \mathrm{~Hz}$ sinusoidal reference with 30 degrees of amplitude, mathematically described by Eqn. (15) as shown in Fig. 5 (solid line)

$$
\theta_{d}=30 \sin \left(\frac{1}{10} t\right)+30
$$

The controlled variable is $\theta_{1}$ which is the angular position of Coupler 1 with respect to $X$ - axis (see Fig. 2). The parameters of the system used for simulation are listed in Tab. I and were taken from manufacturer in [23], [24] and [17], [18].
TABLE I

Simulation PARAMETERS [17], [18], [23], [24]

\begin{tabular}{cllc}
\hline Parameter & Value & Parameter & Value \\
\hline$E_{M}$ & $28 \mathrm{GPa}$ & $C_{A}$ & $10 \mathrm{Mpa} /{ }^{\circ} \mathrm{K}$ \\
$E_{A}$ & $75 \mathrm{GPa}$ & $C_{M}$ & $10 \mathrm{Mpa} /{ }^{\circ} \mathrm{K}$ \\
$A_{s}$ & $88^{\circ} \mathrm{C}$ & $T_{a m b}$ & $25^{\circ} \mathrm{C}$ \\
$A_{f}$ & $98^{\circ} \mathrm{C}$ & $A$ & $4.9 \times 10^{-8} \mathrm{~m}^{2}$ \\
$M_{s}$ & $72^{\circ} \mathrm{C}$ & $A_{w}$ & $290.45 x 10^{-6} \mathrm{~m}^{2}$ \\
$M_{f}$ & $62^{\circ} \mathrm{C}$ & $c_{p}$ & $320 \mathrm{~J} / \mathrm{Kg}^{\circ} \mathrm{C}$ \\
$m_{w}$ & $6.8 x 10^{-4} \mathrm{~kg} / \mathrm{m}$ & $\varepsilon_{L}$ & $2.3 \%$ \\
$R$ & $20 \Omega / m$ & $h_{0}$ & 20 \\
$l_{0}$ & $0.37 \mathrm{~m}$ & $h_{2}$ & 0.001 \\
$b_{s}$ & 0.5 & $b_{1}, b_{2}$ & 0.1 \\
$k_{s}$ & $0.0018 \mathrm{Nm} / 1^{\circ}$ & $\Theta$ & -0.055 \\
\hline
\end{tabular}

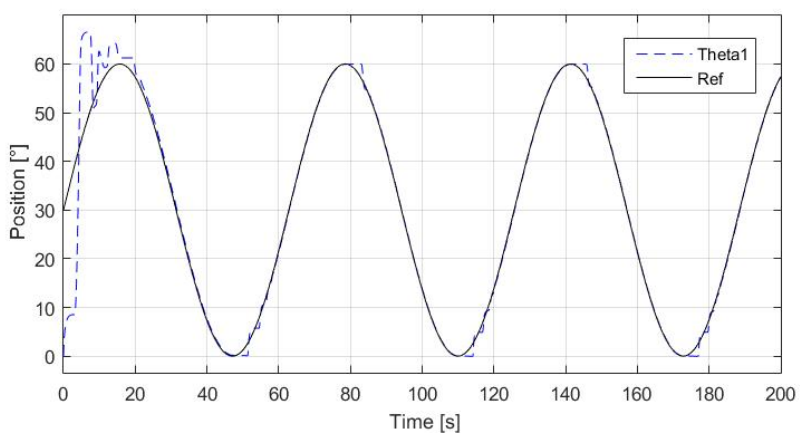

Fig. 5. Tracking response for sinusoidal reference

The sliding mode control was set as follows: The boundary layers $\phi_{1}=10^{\circ}, \phi_{2}=7^{\circ}$ degrees, and voltage constraint $V_{1 H}=6.5 \mathrm{~V}, V_{2 H}=6.5 \mathrm{~V}$. The results of this simulation are shown in Fig. 5. In Fig. 5 it can be seen that the system is not capable of tracking the reference during the first semicycle, it takes around 20 seconds for the system to reach the reference. This is attributed to the sudden increased of the reference during the first cycle. During this period there is a maximum overshoot of $6.6^{\circ}$. After 20 seconds, the system follows the reference with good accuracy, the error oscillates between $-0.2^{\circ}$ and $0.3^{\circ}$ but during the inflection points, as shown in Fig.6. The error increases when the reference goes trough an inflection point, and at these points the error range is between $-2.8^{\circ}$ and $2.5^{\circ}$. This increased error last around 4 seconds, after this transitory period the average tracking error is $\pm 0.3^{\circ}$. Figure 7 illustrates the inputs of the system during the closed-loop test. The inputs are given in Volts and they are limited to avoid thermal damage to the SMA wires, which can destroy its memory effect. Both SMA wires have the same high voltage limit, however, the boundary layer for each wire is set at different levels as mentioned before. The higher limit for $\phi_{1}$ is fixed in order to achieve a faster response from the control law. SMA 2 adjusts the stiffness of the joint. This means that the SMA 2 does not directly actuate over the end effector, and thus its velocity of response is not as critical as SMA 1. In Fig. 7 we can see the rough reaction of the controller during the first cycle of tracking and inflection points. However, after 


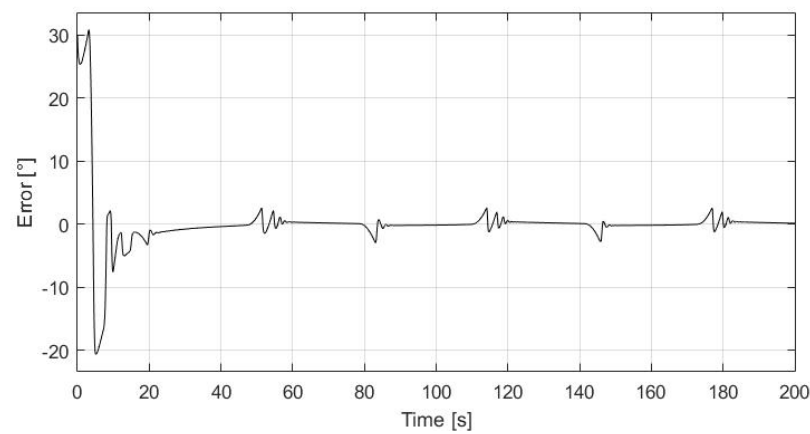

Fig. 6. Angular position tracking error

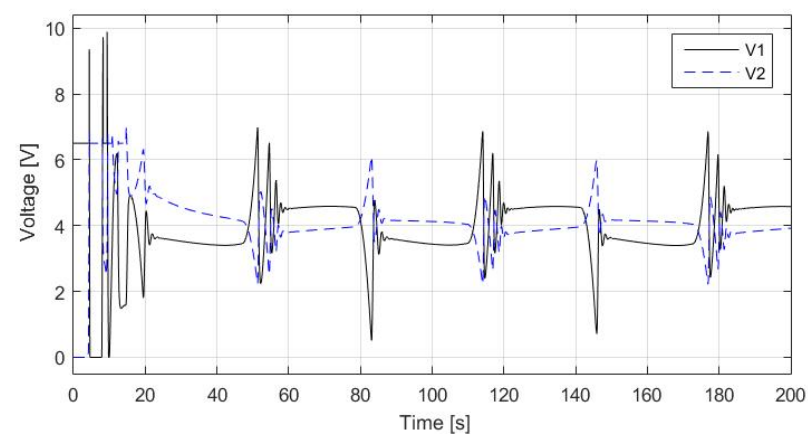

Fig. 7. Voltage input for a sinusoidal reference

settling time of 20 seconds for the first cycle and 4 seconds for the inflection points the control signal is smooth again. This allows a gentle movements of the actuator.

\section{CONCLUSION}

We have presented a 1-DOF SMA wire actuated lightweight robotic arm, which is intended to be an alternative for flying manipulators. This arm is actuated by a couple of antagonistic SMA wires, has a total weight of $48 \mathrm{~g}$ and a range of movement up to $85^{\circ}$ on the $X-Z$ plane. With this weight, our proposal is $75 \%$ lighter than the lightest design found in the literature [7]. In addition the available payload of the robotic arm here presented is around $500 \mathrm{~g}$, which is more than twice the available payload achieved in [7]. However, the lifting capacity of our proposal and can be increased by the use of thicker SMA wires, this will decreased the velocity of response by incrementing the cooling time of the wires, so a compromise between lifting capacity and velocity of response has to be made.

A sliding mode control was applied for angular position tracking. This control approach demonstrated its capability of dealing with the double loop hysteresis present in the system due to the antagonistic SMA wires. An error range between $0.2^{\circ}$ and $0.3^{\circ}$ was achieved except for the reference's inflection points where the error range increase to be between $-2.8^{\circ}$ and $2.5^{\circ}$ for an average period of 4 seconds.
The given SMA based robotic arm is currently under construction. Future work will be orientated to construct and to test experimentally the presented design. In addition, the proposed SMA based robotic arm will be attached to an small quadcopter for flying manipulation analysis. Furthermore an ON/OFF control will be develop for grasping control.

\section{REFERENCES}

[1] T. W. Danko and P. Y. Oh, "A hyper-redundant manipulator for mobile manipulating unmanned aerial vehicles," in Unmanned Aircraft Systems (ICUAS), 2013 International Conference on. IEEE, 2013, pp. 974-981.

[2] C. M. Korpela, T. W. Danko, and P. Y. Oh, "Designing a system for mobile manipulation from an Unmanned Aerial Vehicle," Technologies for Practical Robot Applications (TePRA), 2011 IEEE Conference on, pp. 109-114, 2011.

[3] F. Ruggiero, M. A. Trujillo, R. Cano, H. Ascorbe, A. Viguria, C. Peréz, V. Lippiello, A. Ollero, and B. Siciliano, "A multilayer control for multirotor UAVs equipped with a servo robot arm," IEEE International Conference on Robotics and Automation (ICRA), pp. 4014-4020, 2015.

[4] T. W. Danko and P. Y. Oh, "Design and control of a hyper-redundant manipulator for mobile manipulating unmanned aerial vehicles," Journal of Intelligent \& Robotic Systems, vol. 73, no. 1, pp. 709-723, 2014. [Online]. Available: http://dx.doi.org/10.1007/s10846-013-9935-2

[5] R. Cano, C. Perez, F. Pruano, A. Ollero, and G. Heredia, "Mechanical design of a 6-dof aerial manipulator for assembling bar structures using uavs," in 2nd IFAC Workshop on Research, Education and Development of Unmanned Aerial Systems. IFAC, 2013, pp. -.

[6] A. Suarez, G. Heredia, and A. Ollero, "Lightweight compliant arm for aerial manipulation," in Intelligent Robots and Systems (IROS), 2015 IEEE/RSJ International Conference on, Sept 2015, pp. 1627-1632.

[7] C. D. Bellicoso, L. R. Buonocore, V. Lippiello, and B. Siciliano, "Design, modeling and control of a 5-dof light-weight robot arm for aerial manipulation," in Control and Automation (MED), 2015 23th Mediterranean Conference on, June 2015, pp. 853-858.

[8] F. Nematzadeh and S. Sadrnezhaad, "Effects of material properties on mechanical performance of nitinol stent designed for femoral artery: Finite element analysis," Scientia Iranica, vol. 19, no. 6, pp. $1564-$ 1571, 2012.

[9] X. Liu, Y. Wang, D. Yang, and M. Qi, "The effect of ageing treatment on shape-setting and superelasticity of a nitinol stent," Materials Characterization, vol. 59, no. 4, pp. 402-406, 2008.

[10] N. Pandis and C. P. Bourauel, "Nickel-titanium (niti) arch wires: the clinical significance of super elasticity," in Seminars in Orthodontics, vol. 16, no. 4. Elsevier, 2010, pp. 249-257.

[11] E. A. Williams, G. Shaw, and M. Elahinia, "Control of an automotive shape memory alloy mirror actuator," Mechatronics, vol. 20, no. 5, pp. 527-534, 2010. [Online]. Available: http: //dx.doi.org/10.1016/j.mechatronics.2010.04.002

[12] S. Quintanar-Guzmán, J. Reyes-Reyes, and M. d. c. Arellano-Sánchez, "Modelado y control de un sistema electrotérmico-mecánico móvil basado en alambres musculares," in XVI Congreso Latinoamericano de Control Automtico, CLCA 2014. Asociación de México de Control Automático, 2014, pp. 834-839.

[13] M. Hulea and C. F. Caruntu, "Spiking neural network for controlling the artificial muscles of a humanoid robotic arm," in 2014 18th International Conference on System Theory, Control and Computing, ICSTCC 2014. IEEE, oct 2014, pp. 163-168. [Online]. Available: http: //ieeexplore.ieee.org/lpdocs/epic03/wrapper.htm?arnumber=6982409

[14] A. Khodayari, M. Talari, and M. M. Kheirikhah, "Fuzzy PID controller design for artificial finger based SMA actuators," IEEE International Conference on Fuzzy Systems, pp. 727-732, 2011.

[15] J. Ko, M. B. Jun, G. Gilardi, E. Haslam, and E. J. Park, "Fuzzy PWM-PID control of cocontracting antagonistic shape memory alloy muscle pairs in an artificial finger," Mechatronics, vol. 21, no. 7, pp. 1190-1202, 2011. [Online]. Available: http://dx.doi.org/10.1016/j. mechatronics.2011.07.003

[16] S. Kannan, C. Giraud-Audine, and E. Patoor, "Application of laguerre based adaptive predictive control to shape memory alloy (sma) actuator," $\{$ ISA $\}$ Transactions, vol. 52, no. 4, pp. 469 - 479, 2013. [Online]. Available: http://www.sciencedirect.com/science/article/ pii/S0019057813000293 
[17] M. H. Elahinia and H. Ashrafiuon, "Nonlinear Control of a Shape Memory Alloy Actuated Manipulator," Journal of Vibration and Acoustics, vol. 124, no. 4, p. 566, 2002.

[18] Z. Guo, Y. Pan, L. B. Wee, and H. Yu, "Design and control of a novel compliant differential shape memory alloy actuator," Sensors and Actuators A: Physical, vol. 225, pp. 71-80, apr 2015.

[19] S. Kannan, C. Giraud-Audine, and E. Patoor, "Laguerre model based adaptive control of antagonistic shape memory alloy (sma) actuator," in SPIE Smart Structures and Materials+ Nondestructive Evaluation and Health Monitoring. International Society for Optics and Photonics, 2010, pp. 764 307-764307.

[20] — "Control of shape memory alloy (sma) actuator using seriesparallel model reference adaptive control (mrac)," in ASME 2009 Conference on Smart Materials, Adaptive Structures and Intelligent Systems. American Society of Mechanical Engineers, 2009, pp. 441-450.

[21] S. Kannan, "Modélisation et commande dactionneurs à alliage à mémoire de forme," Ph.D. dissertation, l'École Nationale Supérieure d'Arts et Métiers, 2011.

[22] C. Liang and C. A. Rogers, "One-dimensional thermomechanical constitutive relations for shape memory materials," Journal of intelligent material systems and structures, vol. 1, no. 2, pp. 207-234, 1990.

[23] "Flexinol actuator wire technical and design data," http://www.dynalloy. com/tech_data_wire.php, accessed: 2016-04-26.

[24] "Technical characteristics of flexinol," http://www.dynalloy.com/pdfs/ TCF1140.pdf, accessed: 2016-04-26. 